This item was submitted to Loughborough's Research Repository by the author.

Items in Figshare are protected by copyright, with all rights reserved, unless otherwise indicated.

\title{
Lidocaine-loaded fish scale-nanocellulose biopolymer composite microneedles
}

PLEASE CITE THE PUBLISHED VERSION

http://dx.doi.org/10.1208/s12249-017-0758-5

\section{PUBLISHER}

Springer (?) American Association of Pharmaceutical Scientists

\section{VERSION}

AM (Accepted Manuscript)

\section{PUBLISHER STATEMENT}

This work is made available according to the conditions of the Creative Commons Attribution-NonCommercialNoDerivatives 4.0 International (CC BY-NC-ND 4.0) licence. Full details of this licence are available at: https://creativecommons.org/licenses/by-nc-nd/4.0/

\section{LICENCE}

CC BY-NC-ND 4.0

\section{REPOSITORY RECORD}

Medhi, Pangkhi, Ololade Olatunji, Atul Nayak, Chandrateja Uppuluri, Richard T. Olsson, Buchi N. Nalluri, and Diganta Das. 2017. "Lidocaine-loaded Fish Scale-nanocellulose Biopolymer Composite Microneedles". figshare. https://hdl.handle.net/2134/24679. 


\section{Lidocaine loaded fish scale-nanocellulose biopolymer composite microneedles}

Pangkhi Medhi ${ }^{\mathrm{a}}$, Ololade Olatunji ${ }^{\mathrm{b}}$, Atul Nayak ${ }^{\mathrm{a}}$, Chandra Teja Uppuluric, Richard T Olsson ${ }^{d}$, Buchi N. Nalluric, Diganta B Das ${ }^{\mathrm{a}, *}$

${ }^{a}$ Department of Chemical Engineering, Loughborough University, Loughborough, United Kingdom

${ }^{\mathrm{b}}$ Department of Chemical Engineering, University of Lagos, Akoka, Lagos, Nigeria

c K.V.S.R Siddhartha College of Pharmaceutical Sciences, Pinnamaneni Polyclinic Road, Siddhartha Nagar, Vijayawada, Andhra Pradesh, India

${ }^{\mathrm{d}}$ School of Chemical Science and Engineering, Polymeric Material Division, KTH Royal Institute of Technology, Stockholm, Sweden

\section{Submitted for review and publication in}

\section{AAPS PharmSciTech}

* Corresponding Author (Email: D.B.Das@lboro.ac.uk) 
1

2

3

4

5

6

7

8

9

10

\section{Abstract}

Microneedle (MN) technology has emerged as an effective drug delivery system and it has tremendous potential as a patient friendly substitute for conventional methods for transdermal drug delivery (TDD). In this paper we report on the preparation of lidocaine loaded biodegradable microneedles, which are manufactured from fish scale derived collagen. Lidocaine, a common tissue numbing anaesthetic, is loaded in these microneedles with an aim of delivering the drug with controlled skin permeation. Evaluation of lidocaine permeation in porcine skin has been successfully performed using Franz diffusion cell (FDC) which has shown that the drug permeation rate increases from 2.5 to $7.5 \% \mathrm{w} / \mathrm{w}$ after 36 hours and pseudo steady state profile is observed from 5.0 to $10.0 \% \mathrm{w} / \mathrm{w}$ lidocaine loaded microneedle. Swelling experiments have suggested that the microneedles have negligible swellability which implies that the patch would stick to the tissue when inserted. The experiments on MN dissolution have depicted that the lidocaine loaded in the patch is lower than the theoretical loading, which is expected as there can be losses of the drug during initial process manufacture.

Keywords: Fish scale biopolymer-nanocellulose microneedle, dissolution studies, permeation studies, swelling studies

\section{Introduction}

Lidocaine is a medication used for numbing tissue in a specific area is most commonly delivered as a subcutaneous or nerve targeting injection rather than other administrative measures such as intravenous infusion, nasal spray, etc. The lipophilic nature of lidocaine accounts for the rapid redistribution of lidocaine into peripheral tissue after an intravenous (IV) injection. Injections with hypodermic needles used to deliver lidocaine are important from a clinical standpoint but painful. They may induce hypersensitivity, bruising, discomfort and bleeding at the site of administration, and in some cases, are associated with the risks of contamination (1). Drug-loaded biodegradable microneedle patch is a unique method of transdermal delivery system. It ensures targeted delivery and the waiting time for lidocaine to act on the nerves can be reduced. These are few challenges can be overcome by microneedle assisted transdermal delivery of lidocaine. Normally MN arrays are loaded with the concerned drug and applied on skin surface with a certain amount of pressure to be able to pierce the skin in order to reach beyond the stratum corneum depth. Biodegradable MNs are economical due to the use of fairly cheap materials, reproducible and are generally safe if MN fragments break off after piercing the skin (2). Compared with injectable and skin-surface drug applicants these MNs are beneficial in terms of constant needle lengths being below the known depth causing epidermal pain stimulation during insertion (2). Lidocaine is characterized by rapid onset of action and intermediate duration of efficacy (3). It is also the important class1-b antiarrhythmic drug and administered 
intravenously for the treatment of ventricular arrhythmias (4). Inhaled lidocaine can be used as cough suppressor (antitussive) (3).

Previous studies have proven that consistent and rigid arrays of MNs can be fabricated from a blend of fish scale biopolymer (FSBP) and nanocellulose (NC) (5). These MNs successfully pierce into porcine skin and degrade in the skin. These previous study however did not investigate the ability of the FSBP-NC MNs to load and release drug transdermally. It is important that the MNs are able to penetrate into the skin following drug loading and that the loaded drug is released when inserted into the skin. Keeping this in mind, we aim to investigate the transdermal delivery of lidocaine using biodegradable MNs fabricated from fish scale biopolymer-nanocellulose (FSBP-NC) composites using scales of Tilapia (Oreoschromiss sp) and nanocellulose obtained from bacteria. The lidocaine loaded FSBP-NC MNs are a potentially novel state of the art medical device for minimally invasive local anaesthesia. This also has potential commercial implications in the future when reproducibility is achievable for in-vitro drug delivery of lidocaine. The main objectives of our study are investigating essential pharmaceutics based properties such as swelling, lidocaine permeation past the stratum corneum skin region and the dissolution of the MN array on the skin surface.

\section{Method and Materials}

\section{Materials}

Fish scales of Tilapia are sourced from the local fish traders in Akoka, Lagos, Nigeria. The scales are then transported to the laboratory for further processing. The scales are first washed with tap water to get rid of all debris. When not used immediately the scales are dried in open air at an atmospheric temperature of about $27 \pm 3^{\circ} \mathrm{C}$ and transferred to a plastic jar when completely dried for storage until when needed. Samples of $12 \times 4$ arrays of MNs with a mean weight $2.0 \mathrm{~g}$ is taken as the representative for Franz diffusion cell (FDC) and dissolution studies. FDC with an effective diffusional area of $1.77 \mathrm{~cm}^{2}$ and receptor volume of $14 \mathrm{ml}$ (Orchid Scientific Pvt. Ltd, Maharashtra, India) are set up for lidocaine permeation studies. The dissolution apparatus (DS8000) consists of brown coloured flasks fixed with paddle impellers set at $25 \mathrm{~mm}$ above the flask bottom (Lab India Pvt. Ltd, Maharashtra, India). Polymeric MNs obtained from MITI systems, Seoul, Korea, are solid tapered in shape with a length of 500 microns on a substrate $30 \mathrm{~mm} \times 15 \mathrm{~mm}$ in dimension containing $176 \mathrm{MNs}$ in rectangular arrangement.

\section{Extraction of fish scale biopolymer}

The extraction of biopolymer from fish scales is done according to method presented in our previous works $(5,6)$ and described here briefly. The required amounts of dried fish scales which have been washed and cleaned are treated with $0.6 \% \mathrm{w} / \mathrm{v}$ sodium hydroxide to remove non-collagen residues from 
the fish scales. The scales are allowed to soak in the sodium hydroxide for 1 hour in a conical flask whilst swirling every 15 minutes. The scales are then washed repeatedly in water until the wash water becomes neutral indicating all sodium hydroxide has been removed. The pre-treated scales are then transferred into the stainless steel vessel, a 4.5L pressure vessel (PC-70001, Binatone, and Lagos, Nigeria). Water is then added into the vessel at a ratio of 1 to 3 weights of scales to volume of water. The vessel is then sealed with pressure of $80 \mathrm{kPa}$ and heated up to $80^{\circ} \mathrm{C}\left( \pm 5^{\circ} \mathrm{C}\right)$ to commence extraction of hydrolysed collagen from the scales. After 6 hours the process ends and system is allowed to cool. This is then followed by filtration to separate the solid from the liquid. The liquid is further centrifuged to separate smaller solid residues. The supernatant is then transferred to a beaker where it is heated at $80^{\circ} \mathrm{C}$ to allow the water to evaporate. Once the liquid becomes viscous, it is transferred onto a flat rubber base to dry at room temperature for $24 \mathrm{hrs}$. The dried films are separated from the plate and stored in airtight plastic bags until when needed.

\section{Preparation of bacterial cellulose nanofibers (BC)}

The bacterial cellulose is grown on the surface of a 20 litre glucose solution pre-sterilized and inoculated with Acetobacter xylinum - ATCC 23767. The solution is composed of $400 \mathrm{~g}$ of D-glucose (dextrose, ACS reagent, Sigma-Aldrich), $100 \mathrm{~g}$ of yeast extract (Fluka, CAS 8013-01-2), $23 \mathrm{~g}$ of anhydrous citric acid (reagent grade, Scharlau), $23.8 \mathrm{~g}$ of magnesium sulphate (puriss, p.a., Fluka), $145 \mathrm{~g}$ of triphosphate water from Scharlau, and 1 vol\% ethanol (96\%). The solution is inoculated with a $150 \mathrm{ml}$ fully developed pre-inoculated bacteria culture prepared from the preserved strain in the glass ampoule. The time to generate the uniformly growing cellulose pellicle mats in each pre-inoculated sample is $48 \mathrm{~h}$ at $28^{\circ} \mathrm{C}$. The wet microbial cellulose pellicle mat formed on the surface of the growth medium is weighed after 48 hours ca. $1.0 \mathrm{~kg}$ (including the growth medium inside). The mats are repeatedly boiled in distilled water (5x) until all growth medium is removed and is further boiled twice in 10 vol\% concentrated $\mathrm{NaOH}(2 \mathrm{~L} \times$ 2) for $20 \mathrm{~min}$. The final $\mathrm{pH}$ after all removal of residual chemicals is neutral $(\mathrm{pH} \approx 7)$.

The bacterial cellulose $(\mathrm{BC})$ nanofibers composed of the unique fibrils are extracted from the $1.0 \mathrm{~kg}$ of bacterial cellulose is obtained by shredding the mat using a blender followed by compressing the suspension in a SEFAR polyamide mesh (PA 1000 120/305-35W with $46 \mu \mathrm{m}$ openings) to remove the water. The total mass of wet cellulose is 148 grams. The water remaining in the compressed cellulose is $87.2 \pm 0.8 \%$, corresponding to the dry bacterial cellulose content of $18.9 \mathrm{~g}$. The $148 \mathrm{~g}$ of cellulose is added to a $2 \mathrm{~L}$ aqueous hydro sulphuric acid solution containing 30 vol\% of reagent grade acid (Merck $\mathrm{KGaA})$, and is stirred at $60 \pm 0.5^{\circ} \mathrm{C}(300 \mathrm{rpm})$. The suspension is stirred for 7 hours isothermally. The extraction is terminated by the addition of $2 \mathrm{~L}$ cold MilliQ water and isothermally centrifuged at 10000 $\mathrm{rpm}\left(15^{\circ} \mathrm{C}\right)$ for $10 \mathrm{~min}$, four times. The total amount of extracted CNFs is $10.4 \mathrm{~g}$ (55 wt\% yield), from 148 
$\mathrm{g}$ of shredded cellulose (or $18.9 \mathrm{~g}$ of corresponding dry bacterial cellulose). The specific surface area of the CNFs is calculated to ca. $160 \mathrm{~m}^{2} \mathrm{~g}^{-1}$ from size distributions of a minimum of 500 counted fibrils deposited on TEM grids. Before using in the composites, the BC is prepared as a $28 \mathrm{mg} / \mathrm{ml}$ aqueous suspension.

Production of lidocaine loaded fish scale biopolymer - nanocellulose films (FSBP-NC)

For this study a $15 \% \mathrm{w} / \mathrm{w}$ concentration of NC per polymer weight is used based on previous findings that a higher concentration of NC gave improved mechanical properties and since the key goal of this study is to study the effect of drug loading, the concentration of NC is kept constant. The method which is used to prepare the films is similar to that reported in our previous publication (5) and briefly outlined here. FSBP is weighed and dissolved in distilled water at $45^{\circ} \mathrm{C}$ until all the solid is completely dispersed in water, lidocaine powder is then added at the desired concentration for each formulation, ranging from $0,2.5,5,7.5$ to $10 \% \mathrm{w} / \mathrm{w}$. This is placed under continuous stirring on a magnetic stirrer as the required amount of NC is added drop wise. The mixture is then stirred for 1 hour at $200 \mathrm{rpm}$ at $45^{\circ} \mathrm{C}$ followed by increasing the temperature to $80^{\circ} \mathrm{C}$ until all water gets evaporated. This viscose blend is then transferred onto a silicone plate and allowed to dry at room temperature for 24 hours after which it is placed in airtight plastic bags until needed.

\section{MN Fabrication}

MNs are produced according to method reported in Olatunji and Olsson (5). Silicone elastomer with activator and base mixed in the ratio 1:10 (Bristol Scientific, Lagos, Nigeria) is poured over solid MN moulds and left to cure for $48 \mathrm{hrs}$ at room temperature. The solid mould is then separated from the silicone mould haven formed templates for producing MNs. Films of FSBP-NC-Lidocaine are then cut out in $2 \mathrm{~cm}$ by $2 \mathrm{~cm}$ squares and placed on the mould. This is then placed in between two metal sheets and pressed with approximately $10 \mathrm{~N}$ of force while heating at $80^{\circ} \mathrm{C}$ for 5 minutes. The power is then switched off and the mould transferred to cooling blocks to cool after which the formed MN is separated from the mould Fig. 1. 

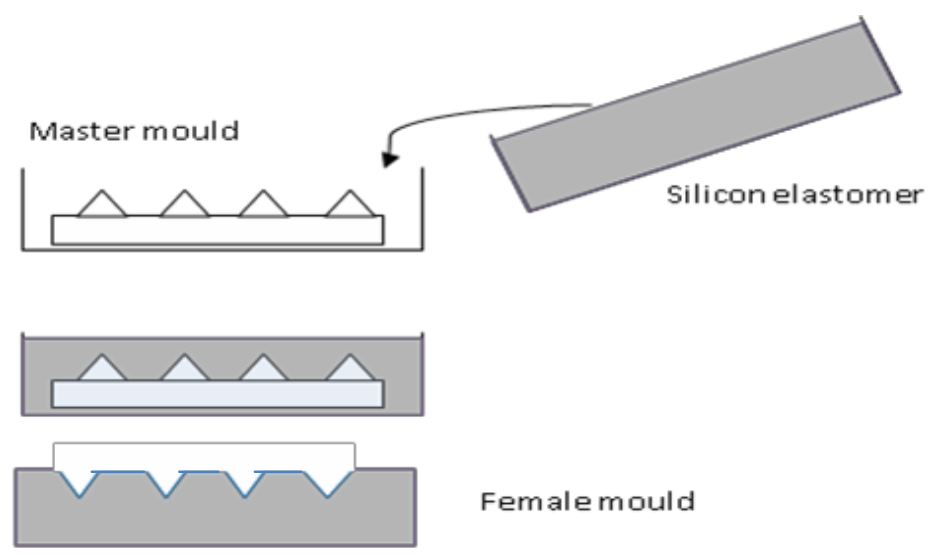

Female mould
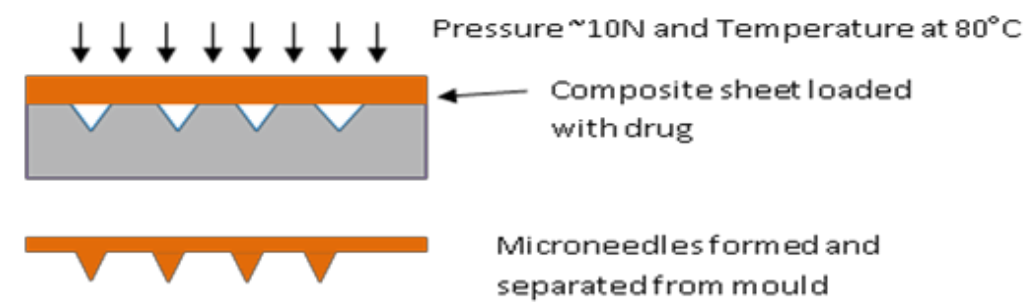

Fig. 1 Schematic diagram of MN fabrication

Swelling studies of fish scale collagen MNs

As these fish biopolymer MNs are biodegradable in nature, swelling studies are performed in order to determine the weight gain due to absorption of moisture from the skin section on application. This is done by measuring the difference in weight of the MN patch. The dry weight of the MN patch is first noted down and then weighed again after its application on the skin. Due to the absorption of moisture from the skin the MN patch shows increase in weight. The increase is however limited to a particular time of application, after which weight decreases with increased time of application due to loss of polymeric mass into the porcine skin. Patch size of approximately $1.5 \mathrm{~cm}^{2}$ is used. Thumb pressure is used to apply the patch onto the skin section. The porcine skin section is treated to achieve physiological temperature of $37^{\circ} \mathrm{C}$. The time of application is variable for each patch. In vitro studies on lidocaine fish scale MNs suggests absorption of moisture (gain weight) and degradation (loss) on application to porcine skin, thus helping in drug permeation.

In vitro studies using porcine skin are performed in triplicate using FDCs as outlined in the Materials which are connected in series are equilibrated to physiological conditions $\left(37.4 \pm 0.5^{\circ} \mathrm{C}\right)$. The $\mathrm{MNs}$ are applied to skin by a firm thumb pressure directed at the middle of the patch. A drug free, sticky tape is carefully placed over the $\mathrm{MN}$ base in order to hold the patch as stationary as possible. The timer is immediately started and receptor volumes $(0.5 \mathrm{ml})$ are removed and subsequently replaced with fresh saline. Each receptor volume is analysed by HPLC. 
A dissolution apparatus as described in the Materials section is set up containing saline solution $(0.9 \%$ $\mathrm{w} / \mathrm{w}$ ) maintained at $32^{\circ} \mathrm{C}$. Each $2.5 \%$ to $7.5 \% \mathrm{w} / \mathrm{w}$ lidocaine loaded $\mathrm{MN}$ patch are pressed on the parafilm for 5-10 sec with thumb pressure and then covered in parafilm exposing only the MNs to the saline solution inside the flask. The covered substrate is bound to a small coin as a "sinker" to weigh the MN patch to the bottom of the flask. The $10 \% \mathrm{w} / \mathrm{w}$ lidocaine loaded MN patch is devoid of any parafilm and thus fixed to a mesh. The parafilm wrap prevents contact of saline solution with the substrate of MN patches. At predetermined periodic intervals, a known volume of saline solution is removed in order to quantify the lidocaine dissolution cumulatively.

In order to confirm if MNs can successfully pierce through the stratum corneum, skin insertion studies have been carried out using method describe by Cheung et al (7). Briefly, the skin samples are treated with MNs followed by application of a $50: 50 \mathrm{v} / \mathrm{v}$ solution of methylene blue. The stained samples are then placed in the cryotome machine for 24 hours after which histological sections are cut and visualized using the microscope.

\section{Results}

Microstructural nanocomposite characteristics:

The blends of FSBP/NC are observed using transmission and scanning electron microscopy (TEM/SEM). The TEM and SEM images obtained are shown in Fig. $2 a$ while $2 b$, respectively. Fig. 2a shows the micrographs of the obtained fibres before preparing composites. Fig. $2 b$ shows the cross-sectional scanning electron microscope (SEM) images obtained by using a Hitachi FE-SEM S-4800 microscope after sputtering the composites 20 seconds by a platinum/palladium coating (applied in a Cressington 208HR sputter). The nanocellulose (NC) suspension used shows good dispersion of the nanocellulose fibres in water as the individual cellulose fibres are evenly dispersed in the suspension. Fig. $2 \mathrm{~b}$ shows the dispersion of the (NC) fibres within the FSBP matrix. The bacteria cellulose in this case shows very high compatibility with the fish scale biopolymer. The NC fibres retained their individual length and diameter and in most of the formulation the NC exists as individual fibres rather than clustered. 
176 tips which replicate the structure of the master moulds used. The MNs formed from the master moulds 177 are cut into approximately equal sizes by length and width. Each array contained 48 MNs arranged in a 17812 by 4 pattern as shown in Fig. 3.

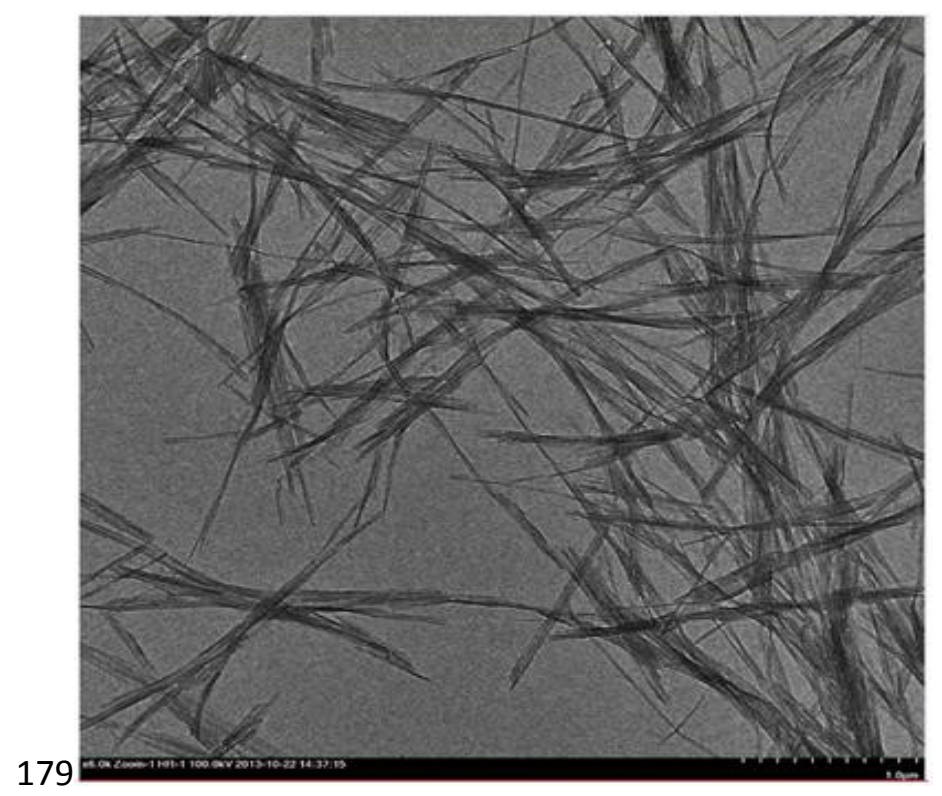

180 Fig. 2a. TEM showing cellulose microfibrils before preparing a composite.
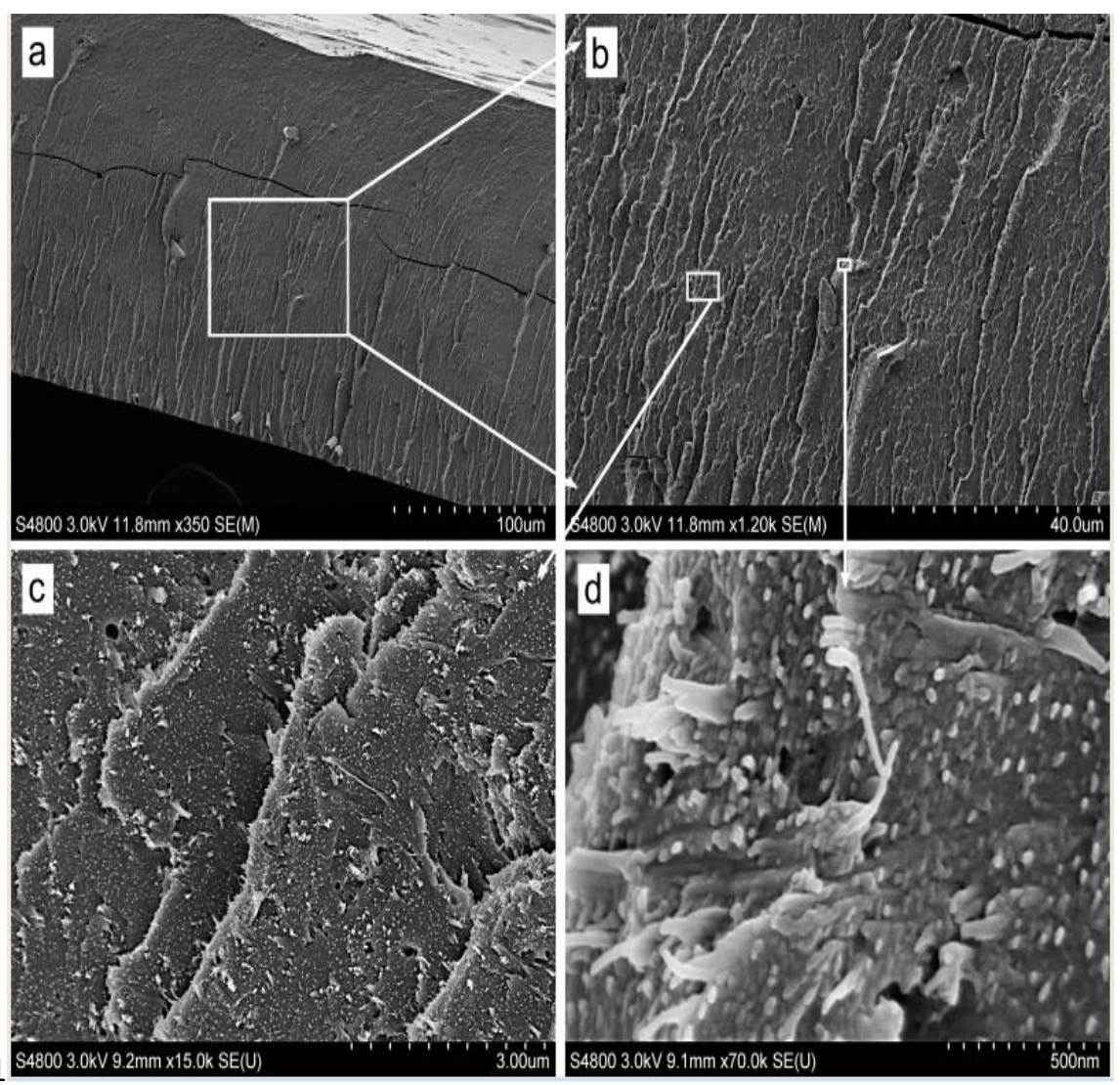

Fig. 2b. HR-SEM showing FSBP-NC composite at 350x, 12000x, 15000x and 70000x resolutions 


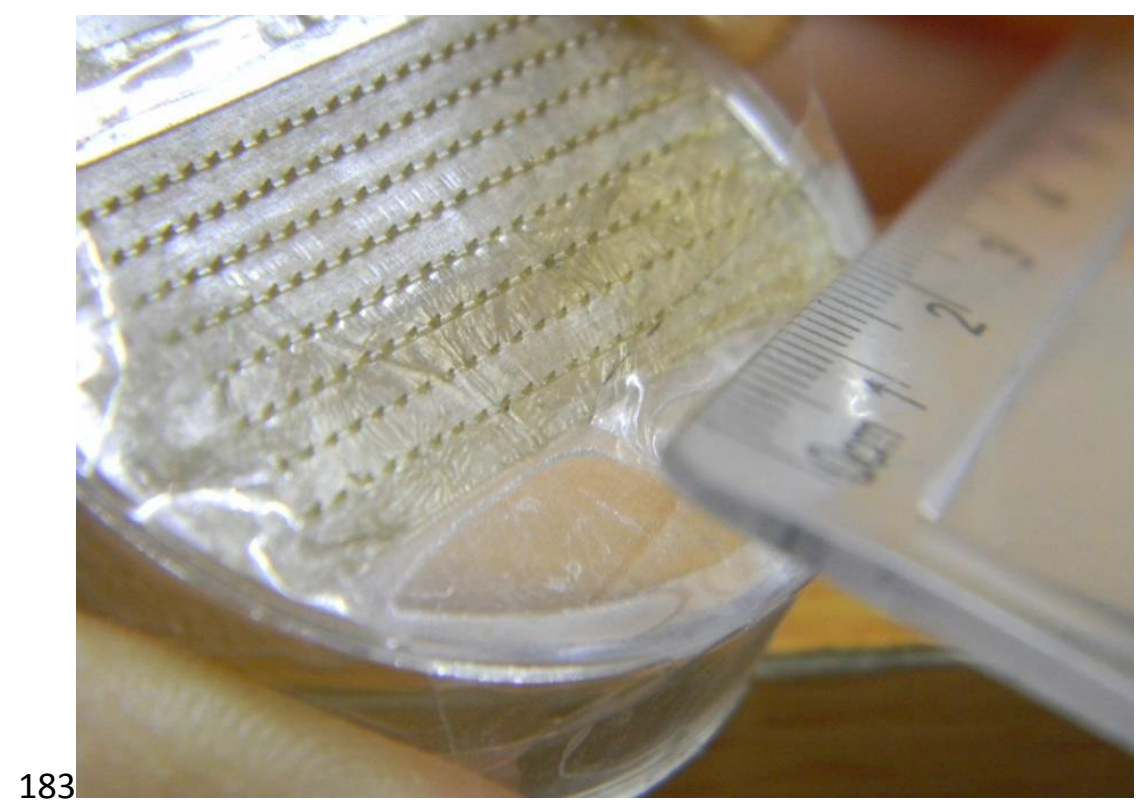

184 Fig. 3. Fish scale biopolymer/nanocellulose $\mathrm{MN}$ array containing Lidocaine insertion studies using 185 cryotome

186 Fig. 4 shows the cavity created by a MN piercing on a porcine transverse skin section made by cryotome.

187 The blue staining on the image indicates the methylene blue stained stratum corneum. From the image 188 the outline of the region pierce by the MN can be seen (marked by red circle). Thus indicating that the 189 MNs are able to pierce through the stratum corneum layer and reach the lower layer of the epidermis.

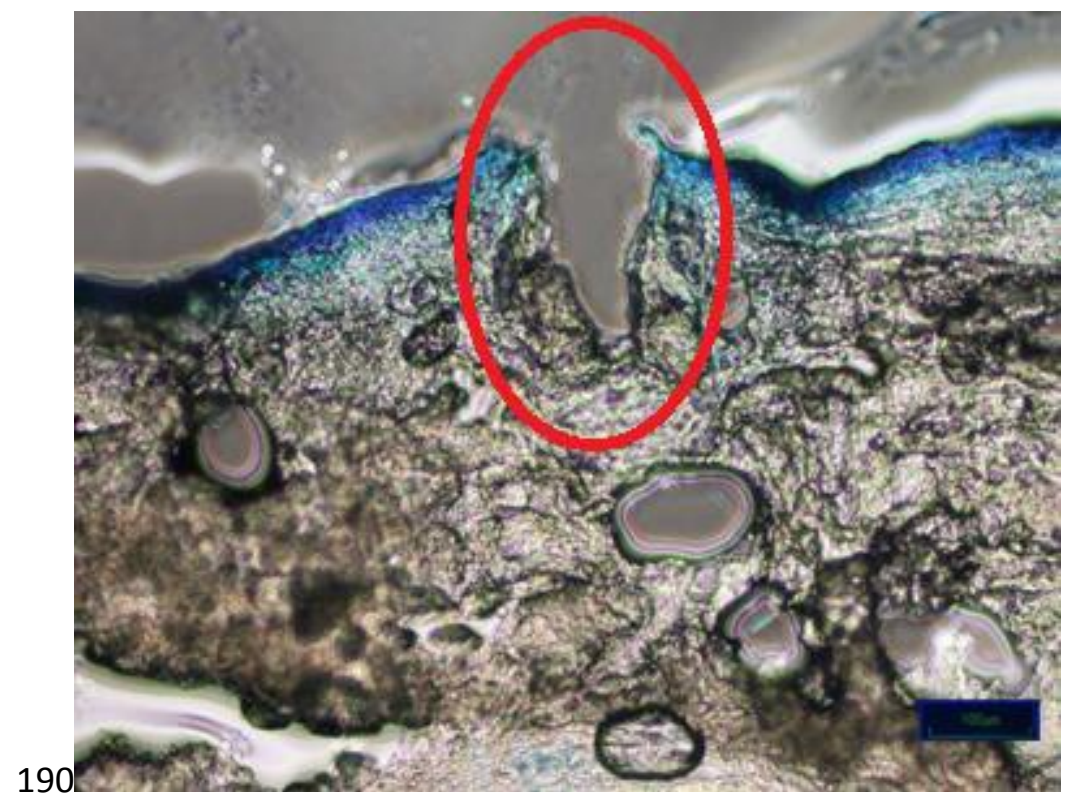

191 Fig. 4a. Cryotome image of skin section showing MN piercing. 
The lidocaine permeation profiles show an increasing trend with time for all four loading concentrations (2.5-10\% w/w) (Fig 5). The error bars (SEM) indicate good reproducibility of pooled data up to 36 hours for all loading concentrations (Fig. 5). A one-tailed F-test was performed to compare the significance of variation between data points representing flux at $t=42$ hours (Fig. 54). The chosen independent time point is the representative of the slope gradient representing the permeation flux against lidocaine loading (Fig. 65). The $F_{2,2}=19.00$ is the accepted critical value $(P=0.05)$. The calculated F-test values varied from 3.60 to 1.14 with comparing $2.5 \%$ till $10 \%$ of lidocaine loading. As F-test values were less than the accepted critical value, the null hypothesis is retained and the variation between lidocaine permeation representatives of flux is insignificant. Nevertheless from first inspection there is visible separation of data points at $t=42$ hours and a slight significance in variation (Fig. 54). Appearance of a rate limiting step is observed within an 18 hour period for lidocaine 2.5, 5 and $10 \% \mathrm{w} / \mathrm{w}$ loading (Fig. 5). This distinct rate limiting observation indicates that most of the drug is in the process of diffusing slowly through the skin so the lidocaine permeated amount is less in the receptor of FDC. The lidocaine $7.5 \%$ $\mathrm{w} / \mathrm{w} \mathrm{MN}$ shows faster permeation within $30 \mathrm{hrs}$ compared to the others, especially $10 \% \mathrm{w} / \mathrm{w}$.

Pseudo steady state profile is observed for 5.0 - $10.0 \% \mathrm{w} / \mathrm{w}$ lidocaine MN loading after 30 hours (Fig. 6). The apparent flux shows increasing trends for $2.5-7.5 \% \mathrm{w} / \mathrm{w}$ after 36 hours.

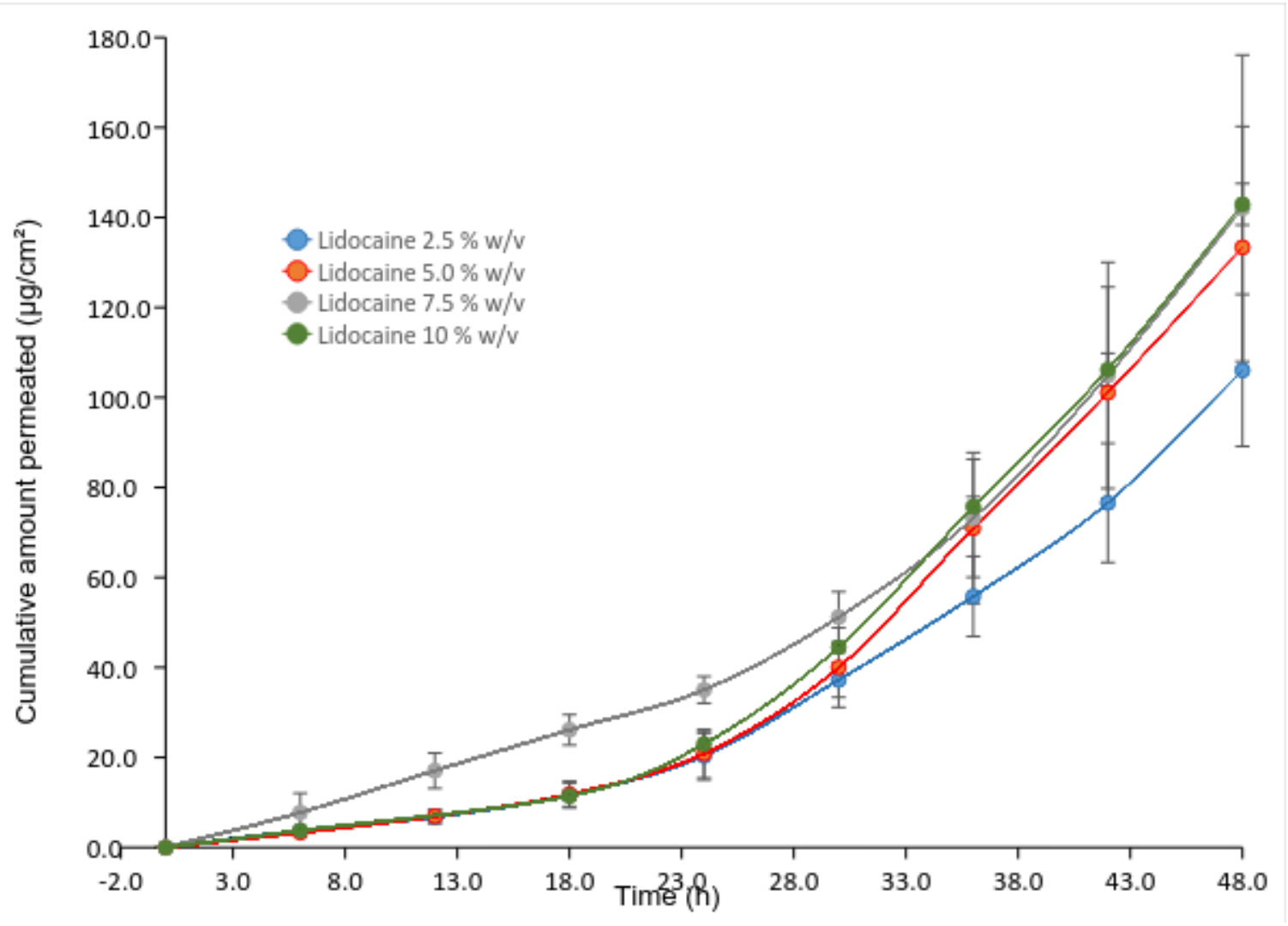

Fig. 5. The permeation of lidocaine through full thickness skin from loaded MNs (Error bars represent the standard error of mean SEM). 


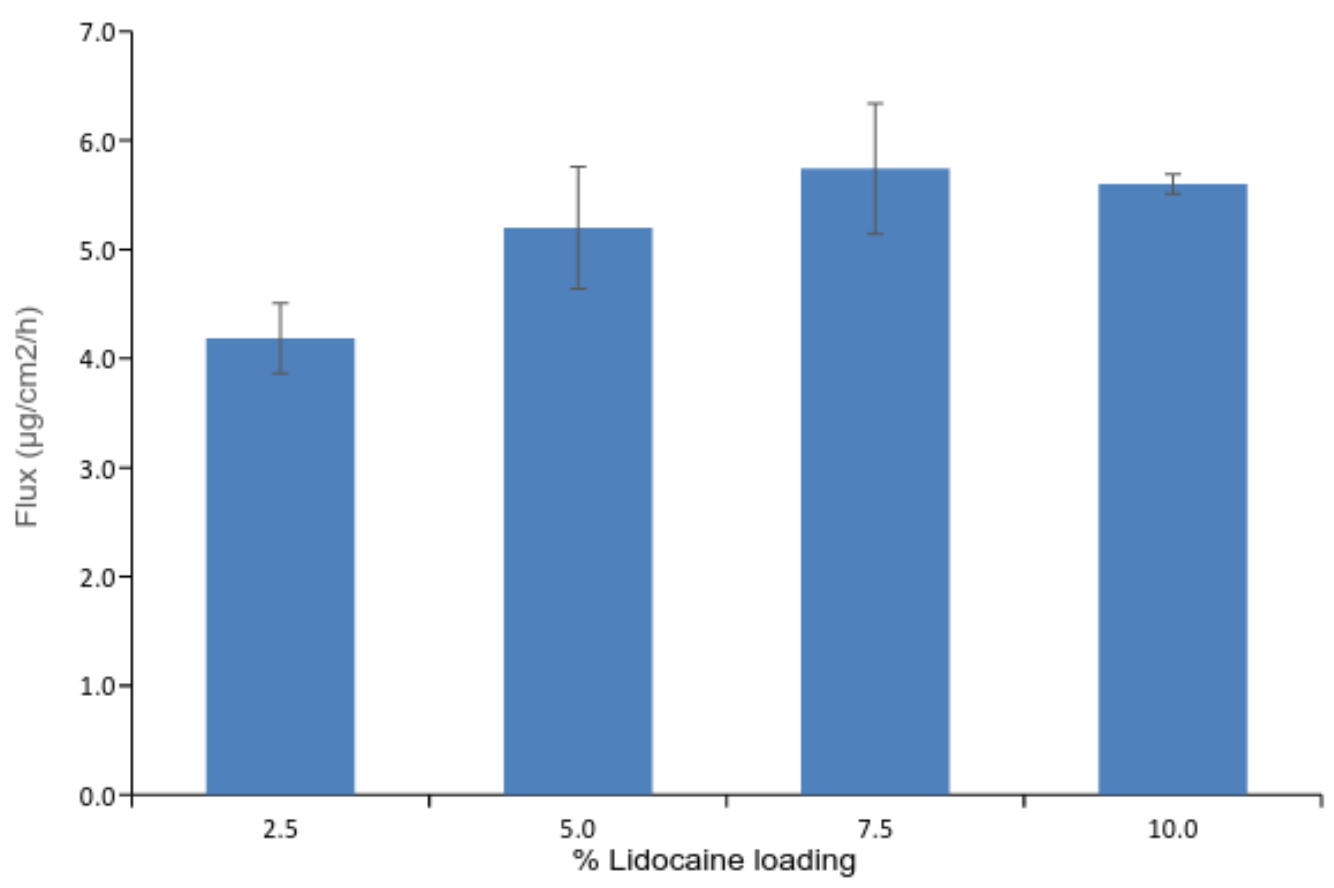

212

213 Fig. 6. The permeation fluxes of lidocaine after a period of 36 hours through full thickness skin from 214 loaded MNs (Error bars represent SEM).

215 Swelling studies

216 The swelling studies are performed to analyse the swellability of these collagen MNs, which will give a

217 fair interpretation of how well they absorb moisture from the tissue and reach the maximum swelling 218 point in order to deliver drug into the system. We took MN insertion time as one of the variable factors 219 into consideration (keeping other factors like pressure applied and size of the MN patch constant) while 220 observing swelling properties.

221 From the experiments we have found that the MN patches have taken around 50 to 55 seconds to reach 222 the maximum swelling point. Maximum mean \% change in mass is $1.12 \%$ (Fig. 7). After which they show 223 degradation and thus loss of mass. It is also observed that the extent of swelling is not very high which 224 ensured the patch to stick to the tissue surface and hence preventing it from falling out. 


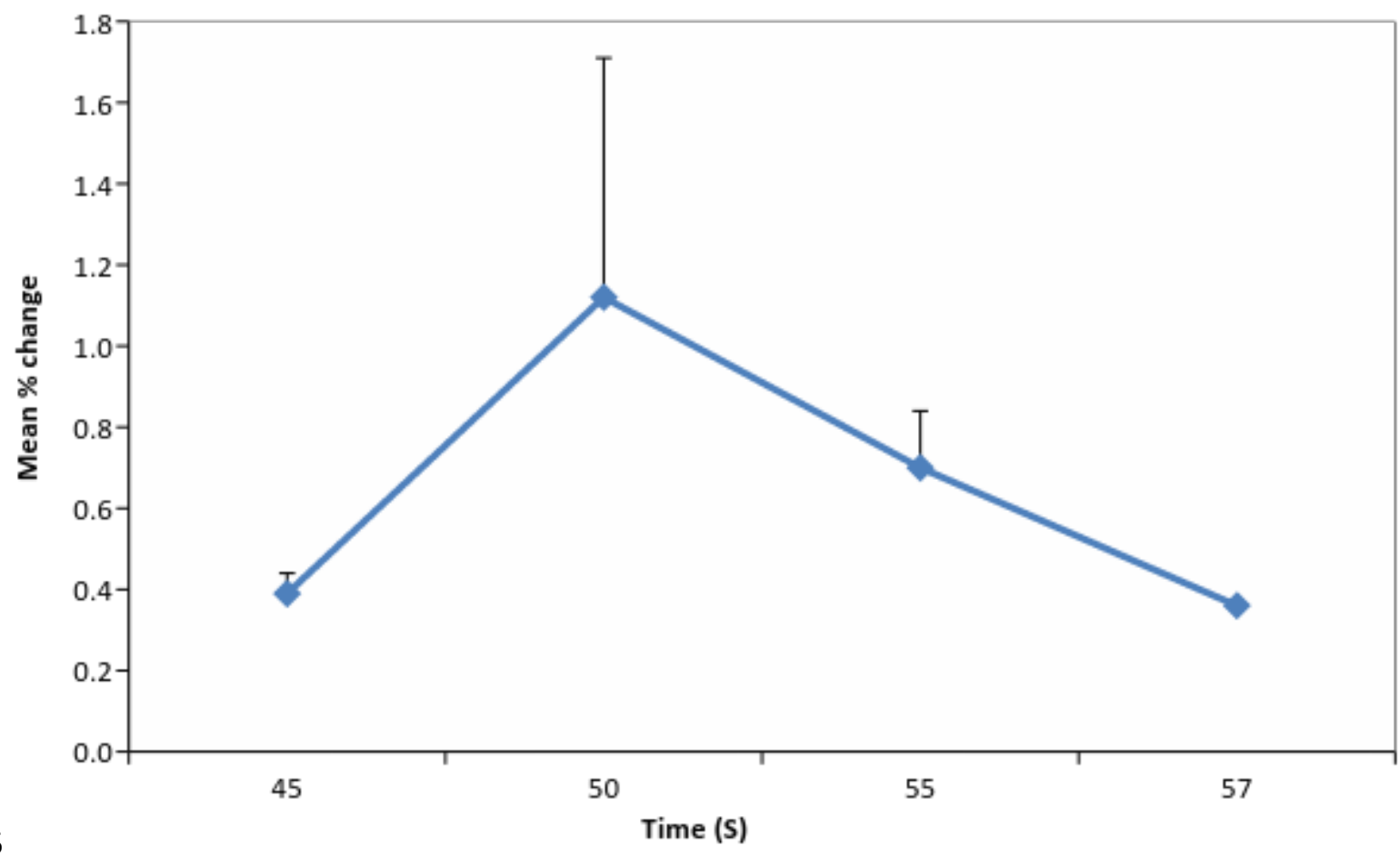

Fig. 7. Change in mass of lidocaine MN patches (Error bars represent SD).

In order to determine the experimental lidocaine loading in MNs from theoretical loading (2.5-10.0\% $w / w)$, dissolution studies allow the complete dissolution of drug molecules in excess solvent. The dissolution profile outlines rapid lidocaine release for $7.5 \%$ and $10 \% \mathrm{w} / \mathrm{w}$ loading (Fig. 8). The highest plateau values on the hyperbolic shaped curve represent the cumulative amount of lidocaine release in dissolution media. The $10 \% \mathrm{w} / \mathrm{w}$ lidocaine loaded MN underwent full dissolution including the substrate so therefore very high fold values are reported here. The theoretical loading mass ranges of lidocaine are from 5 to $22 \mathrm{mg}$ for 2.5 to $10.0 \% \mathrm{w} / \mathrm{w}$ in $2 \mathrm{~g}$ (nanocellulose 15\% of FSBP) of total material content required to make 1 batch of 480 MNs. From the plateau obtained from dissolution profiles, the experimental loading mass of lidocaine in the MNs is $0.3 \mathrm{mg}$ for 2.5 and $5.0 \% \mathrm{w} / \mathrm{w}$ theoretical loading (Fig. 8). The experimental loading mass for $7.5 \% \mathrm{w} / \mathrm{w} \mathrm{MN}$ is $1.6 \mathrm{mg}$ and for the whole $\mathrm{MN}$ including substrate is $7.8 \mathrm{mg}$ for $10 \% \mathrm{w} / \mathrm{w}$ MN (Fig. 8). The percentage loading of lidocaine is calculated per MN batch of $480 \mathrm{MNs}$. Therefore the practical percentage loading is $0.3 \%, 0.3 \%, 1.5 \%$ and $7.5 \%$ lidocaine from 2.5 to $10 \% \mathrm{w} / \mathrm{w}$ theoretical loading of MNs. The lidocaine loading is low for the needle body as examined for 2.5 to $7.5 \% \mathrm{w} / \mathrm{w}$ theoretical loading. Lidocaine is not a very low potent and highly expensive drug so low percentage loading could be permissible in further developmental studies. Nevertheless a target of up to $35 \%$ lidocaine loading is ideal for the needle body itself. At micron scales 
245 preventing lidocaine building up into the substrate is looked into.

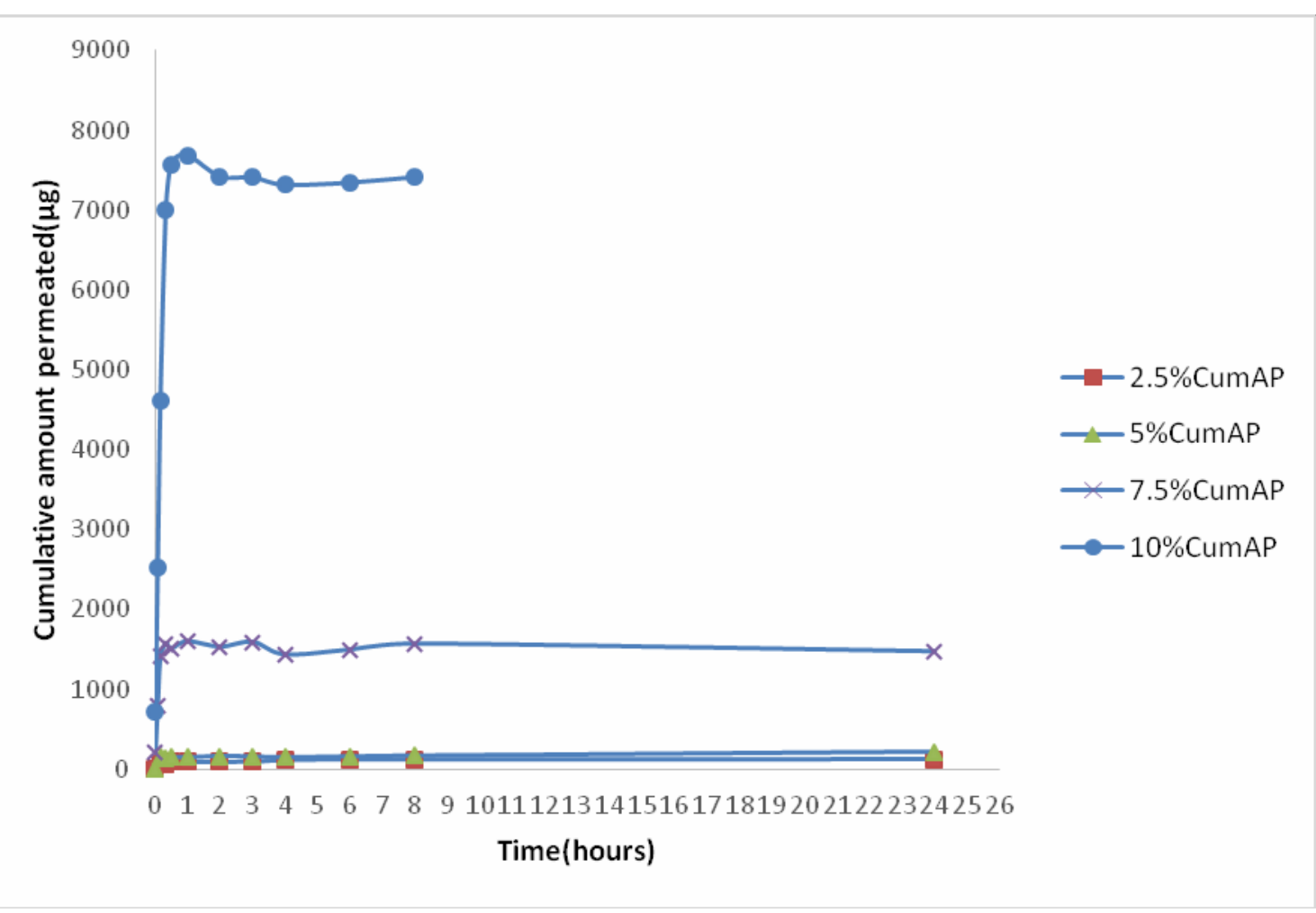

Fig. 8. Dissolution studies of $2.5 \%, 5 \%, 7.5 \%, 10 \%$ lidocaine loaded MNs.

\section{Discussion}

The ability of biopolymer extracted from fish scale to form uniform blend with cellulose particles can be attributed to the protein-polysaccharide intermolecular bonding due to the presence of the amide linkage in protein of the fish scale which is essentially hydrolysed collagen and the $\mathrm{OH}$ group of the cellulose polysaccharide. These forces allow good compatibility ion protein polysaccharide composites (8). Both DSC and FTIR studies are not conducted because the aim of this study was the lidocaine release from microneedle polymeric composite. This shall be performed in a furtherstudy. The flexibility of the gelatine combined with the structural strength of the cellulose resulted in a composite material with sufficient flexibility for micromoulding and a resulting $\mathrm{MN}$ structure with sufficiently sharp and rigid tips. From the results of the skin insertion studies using cryotome it can be seen that the MNs successfully pierced the stratum corneum layer. This is an essential requirement for MNs as they must be able to pierce the stratum corneum in their dry state such that they maintain their structural integrity in the process of piercing the stratum corneum layer. Other studies in literature have also used similar techniques with porcine skin to determine the ability of MNs to pierce the skin $(7,9)$. 
The reproducibility of the fabrication process is also important as it allows formation of microstructures with consistent dimensions.

During the drug loading step of lidocaine in collagen we have assumed a well-mixed system as the drug is dissolved in vehicle itself. Lidocaine is relatively inexpensive and quite potent so the drug is allowed to appear in the base substrate of the MN body. Despite allowing lidocaine to accumulate in the base substrate, there is no contradictory trend in lidocaine flux from $2.5 \% \mathrm{w} / \mathrm{w}$ to $7.5 \% \mathrm{w} / \mathrm{w}$ loading. The tertiary amide groups of lidocaine seem less ionically attracting the collagen amino groups for binding. Fundamentally this physicochemical property for binding lidocaine to fish collagen is less likely to be stronger at $7.5 \% \mathrm{w} / \mathrm{w}$ as compared with others, and therefore faster initial release of lidocaine in skin plasma. This result provide significant evidence that lidocaine loaded MNs made from FSBP-NC composite can act as delivery systems to administer lidocaine transdermally.

In this particular study the MNs did not demonstrate large swelling capability compared to the hydrogel MNs reported in literature (9). As the results from the permeation studies show, despite not showing significant swelling the MNs are able to release loaded drugs into the skin. Rather than the mechanism of absorbing water and swelling, due to the adhesive nature of the gelatine, the MNs in this case insert into the skin and absorb moisture which allows the drug polymer matrix to dissociate thereby releasing the drug from the polymer materials. The $\mathrm{MN}$ material in this case comprises of protein and polysaccharide material which are both biocompatible naturally occurring polymer such that dissociation into the skin pose no health hazard.

The dissolution studies show that the lidocaine loaded into the MNs is released in distilled water within an hour which indicates that the lidocaine loaded FSBP-NC MN can act as medium to fast release MNs. The release did not occur in seconds therefore they do not apply as rapid release polymeric MNs. Further studies will explore the dose dependent release rate in more details.

\section{Conclusion}

The findings of this study provide evidence that $\mathrm{MN}$ arrays produced from fish scale biopolymernanocellulose blends loaded with lidocaine can pierce the stratum corneum and dissolve in the skin to release the loaded drug. In particular the MNs with loaded lidocaine $7.5 \% \mathrm{w} / \mathrm{w}$ outline a favourable permeation of the drug through skin. Also, the drug loading is considerably higher as dissolution studies have shown a higher release for patches loaded with $5.0 \%$ lidocaine than those loaded with $2.5 \%$ w/w lidocaine. Logically the greater the drug loading, the greater the drug release as long as the drug carrying vehicle is not saturated. Further studies will need to explore optimizing drug release rate by varying the formulation of the biopolymer material. 
295 The authors wish to acknowledge the British Council, UK and Directorate of Science and Technology

296 (DST), India for supporting the work. This research is supported by the International Foundation for 297 Science IFS (Grant No. F/5544-1).

\section{References}

299 1. Ita K. Transdermal delivery of drugs with microneedles:Potential and Challenges. Pharmaceutics. $300 \quad 2015 ; 7(3): 90-105$.

3012 2. Nayak A, Das DB. Potential of biodegradable microneedles as a transdermal delivery vehicle for lidocaine. Biotechnol Lett. 2013;35:1351-63.

3. Gordh T. Lidocaine: the origin of a modern local anesthetic. 1949. Anesthesiology . 304

5. Olatunji O, Olsson RT. Microneedles from fishscale-nanocellulose blends using low temperature

6. Olatunji O, Igwe CC, Ahmed AS, Alhassan DOA, Asieba GO, Das DB. Microneedles from fish scale biopolymer. J Appl Polym Sci. 2014;131(12):1-10.

7. Cheung K, Han T, Das DB. Effect of Force of Microneedle Insertion on the Permeability of Insulin in Skin. J Diabetes Sci Technol [Internet]. 2014;8(3):444-52.

8. Sarika PR, Cinthya K, Jayakrishnan A, Anilkumar PR, James NR. Modified gum arabic cross-linked gelatin scaffold for biomedical applications. Mater Sci Eng C [Internet]. 2014;43:272-9.

9. Donnelly RF, McCrudden MTC, Alkilani AZ, Larraneta E, McAlister E, Courtenay AJ, et al. Hydrogelforming microneedles prepared from "super swelling" polymers combined with lyophilised wafers for transdermal drug delivery. PLoS One. 2014;9(10). 